\title{
Motivational Properties of Oxytocin in the Conditioned Place Preference Paradigm
}

Israel Liberzon, M.D., Keith A. Trujillo, Ph.D., Huda Akil, Ph.D., and Elizabeth A. Young, M.D.

We hypothesized that oxytocin might have intrinsic reinforcing properties and studied it using a conditioned place preference. Three studies examining motivational properties of oxytocin in nonpreferred, preferred, and balance designs were performed utilizing two compartment apparatus. On alternate days, compartments were paired with subcutaneously injected oxytocin $(6 \mathrm{mg} / \mathrm{kg})$ or saline, and animal pre- and post-conditioning place preference was compared. Whereas in animals paired with saline there was a shift to a lack of preference, oxytocin-treated animals reversed their preference, spending more time in a previously unpreferred, compartment. In preferred compartment design, oxytocin-treated animals further increased their preference, whereas saline-treated animals decreased their preference toward a nonpreference for either compartment. Our results demonstrate that oxytocin produces a reliable and robust preference for the environment with which it is repeatedly associated, and has rewarding or potentially anti-aversive properties. Future studies are needed to distinguish among these possibilities. [Neuropsychopharmacology 17:353-359, 1997] (C) 1997 American College of Neuropsychopharmacology. Published by Elsevier Science Inc.
KEY WORDS: Reinforcing; Conditioned place preference; Oxytocin; Rats.

In recent years it has been recognized that the hypothalamic nonapeptide oxytocin has an important function as neurotransmitter/neuromodulator within the central nervous system (CNS), in addition to its systemic role as a hormone involved in parturition and lactation. The majority of oxytocin immunoreactive neurons are localized in hypothalamic paraventricular and supraoptic nuclei, and project to multiple sites within the limbic system (Buijs 1978; Buijs and Swaab 1979). High densities of oxytocin receptors are found in the olfactory nu-

From the Mental Health Research Institute, Department of Psychiatry (IL, HA, EAY), University of Michigan, Ann Arbor, MI; the Psychiatry Service (IL), Veterans Administration Medical Center, Ann Arbor, MI; and the Psychology Program, KAT California State University, San Marcos, CA.

Address correspondence to: Israel Liberzon, M.D., Mental Health Research Institute, Department of Psychiatry, University of Michigan, 205 Zina Pitcher Place, Ann Arbor, MI 48109-0720.

Received November 26, 1996; revised March 27, 1997; accepted April 30, 1997. clei, nucleus accumbens, bed nucleus of stria terminalis, ventromedial hypothalamus, lateral septum, amygdala, hippocampus, and subiculum (Tribollet et al. 1988; Tribollet et al. 1992). Behavioral studies suggest that oxytocin is involved in the mediation of a variety of biologically important functions-reproduction, affiliation, ingestion, neuroadaptation, stress regulation, and memory (Burbach et al. 1992; Caldwell 1992; Carter et al. 1992; de Wied 1978; Grassi and Drago 1993; Insel and Shapiro 1992a; Insel and Shapiro 1992b; Kovacs et al. 1979; Panksepp 1992), functions that have been linked to activation of limbic and paralimbic systems.

To date, oxytocin's role in the mediation of reproductive behaviors has been studied the most, and has been established across species in a variety of experimental paradigms. Exogenous administration of oxytocin reliably induces sexual, maternal, and affiliative behavior (Caldwell 1992; Carter et al. 1992; Insel and Shapiro 1992b; Keverne and Kendrick 1992), while oxytocin antagonists or antisera suppress the expression of these behaviors (Insel and Winslow 1991). Other behavioral functions of oxytocin that have been extensively inves- 
tigated are its effects on memory, learning, and neuroadaptation. In studies of learning and memory, oxytocin has been reported to facilitate extinction of learned avoidance (de Wied et al. 1987), while oxytocin antisera have been found to increase retention of passive avoidance (de Wied et al. 1991), suggesting that oxytocin has an intrinsic amnestic property. Oxytocin's effects on memory in humans (Leckman et al. 1994; Mazurek et al. 1987), and its inhibitory effects on the development of dependence and tolerance to opiates and cocaine (Sarnyai and Kovacs 1993) also suggest that it plays an important role in modulation of neuroadaptation and learning (possibly inhibiting these processes). Preliminary data on oxytocin's effects on the stress response, grooming behavior and nociception suggest that oxytocin administration promotes coping with stress by inhibiting some processes and inducing others. Oxytocin decreases distress vocalization, induced by maternal separation, in chicks (Panksepp 1992), diminishes endocrine stress responses (Lightman 1992), prevents formation of stress-induced gastric lesions (Grassi and Drago 1993), and induces stress adaptive behaviors such as grooming (Van Erp et al. 1993; van Erp et al. 1995) and increased tolerance of nociceptive stimuli (Agren et al. 1995).

Although it is possible that each of the above mentioned effects has a separate underlying neurobiological mechanism for its expression and that oxytocin plays a distinct role in each one of these, its seems logical to hypothesize that oxytocin may produce a more general behavioral effect which modifies the expression of these specific behaviors. A possible candidate for this "more general effect" is the presence of reinforcing properties of oxytocin. Such properties would allow this peptide to influence a number of different behaviors. We therefore chose to study motivational properties of oxytocin using a condition place preference paradigm (CPP).

CPP has been used widely in recent years to assess the motivational or reinforcing properties of pharmacological agents (Bardo et al. 1995). Briefly, animals are repeatedly exposed to two different compartments with distinctive cues, one is pared with a drug and the other with the vehicle. If the drug has reinforcing properties, the compartmental cues acquire secondary reinforcing characteristics which result in the animal spending more time in the drug-paired compartment when given a choice between compartments. If the drug has aversive properties, the animal spends less time in the drugpaired compartment. The effect is usually defined by the change in duration spent in the drug-paired compartment after conditioning. While reinforcing properties of drugs are most commonly studied using CPP, the paradigm has also been shown to be useful in studying rewarding or aversive elements of specific behaviors such as sexual interaction (Oldenburger et al. 1992), attenuated reward sensitivity in anhedonia (Papp et al. 1991) and analgesic properties of a given drug
(Sufka 1994), suggesting that CPP is sensitive to changes in general motivational milieu (reinforcing vs. aversive) associated with a given condition. Therefore, if oxytocin has intrinsic reinforcing or aversive properties, one should be able to demonstrate them using the CPP paradigm.

\section{METHODS}

We used a total of 45 experimentally naive, male SpragueDawley rats (Charles River) in three studies $(12,12$, and 21 animals, respectively). Animals were housed in groups of three in stainless steel cages on $12 \mathrm{~h}$ lightdark cycle. Food and water were allowed ad libitum. All animals were allowed 7 days to habituate to the animal colony before the beginning of the experiments (first day of the handling). Animals weighed $250-300 \mathrm{~g}$ at the start of the studies.

\section{Apparatus}

Two identical Plexiglas shuttle boxes $(80 \times 25 \times 50 \mathrm{~cm})$ were used for the experiments. Both compartments had a clear ceiling, stainless steel grid floor and sawdust litter below the floor. One of the compartments had black walls, while the other had white walls. A stainless steel guillotine door separated the compartments and could be removed to allow free access to both compartments. Four photoelectric sensors mounted $1 \mathrm{~cm}$ above the floor, at $5 \mathrm{~cm}$ intervals across the length of each compartment, detected the animal's entrance, presence, and movement, as well as the amount of time spent in the compartment. During the experiment, low intensity white noise was delivered to the testing room to minimize the effects of ambient sounds. The testing rooms were dimly lit by a single ceiling fixture.

We pretested the apparatus for its ability to detect place preference conditioning with two different groups of animals, utilizing morphine sulfate and naloxone as reinforcing and aversive agents, respectively. We could clearly induce both conditioned place preference by morphine sulfate and place aversion by naloxone (Liberzon unpublished data), replicating results published in the literature (Trujillo et al. 1991) regarding the reinforcing properties of these compounds, and validating the testing conditions.

\section{Procedure}

Three separate experiments were performed, using procedures similar to those described previously (Trujillo et al. 1991). Animals were weighed and handled daily for six days prior to testing. During the next three days animals were placed in the apparatus for $15 \mathrm{~min} /$ day with free access to both compartments (guillotine door removed). Animals were placed between the compart- 
ments facing either the white or black compartment. The direction of initial placement (animal head facing white or black compartment) alternated on conditioning days and was counterbalanced within the groups. The amount of time spent by the animal in each compartment during the third (and the last) day of testing was recorded and used as a measure of initial preference. The following eight days served as conditioning days. On alternate days animals were injected subcutaneously with oxytocin and confined to one of the compartments for $30 \mathrm{~min}$, or injected with the saline and confined to the other compartment. The order of the injections was counterbalanced. Oxytocin was paired with the non-preferred compartment in Study I, the preferred compartment in Study II, and there was a random assignment of drug/compartment pairs (balanced design) in Study III. Control animals received saline in both compartments. On the last day (post-conditioning test day), the animals were placed between compartments and allowed free access to both sides for a period of $15 \mathrm{~min}$. The time spent in each compartment was recorded as the post-conditioning place preference. The compartments were cleaned with dilute soap solution and the bedding was changed prior to each animal's exposure, to minimize the odor of the previous animal.

\section{Drug}

We compared oxytocin (Peninsula Labs) to vehicle $(0.9 \%$ $\mathrm{NaCl}$ ). Both oxytocin and saline were injected subcutaneously, using a $25 \mathrm{G}$ needle in a volume of $0.5 \mathrm{ml} \mathrm{im}-$ mediately prior to the placement of the animal in the appropriate compartment. Animals exhibited minimal to no visible distress as a result of injections. Oxytocin was administered at the dose of $6 \mathrm{mg} / \mathrm{kg}$ in Studies I and II and $6 \mathrm{mg} / \mathrm{kg}$ and $0.06 \mathrm{mg} / \mathrm{kg}$ in Study III. Doses were chosen based on the dose ranges used in previously published research, in other behavioral paradigms (Arletti et al. 1990).

\section{Data Analysis}

Motivational properties of oxytocin were determined by its ability to modify the preference of the rat for the compartment paired with peptide. The place preference (dependent measure) is expressed as the difference between the amount of time spent in the drug-paired compartment and the amount of time spent in the saline-paired compartment. This method of calculating place preference has been widely used in conditioned place preference research (Trujillo et al. 1991). Pre-conditioned (initial) preference and post-conditioned (final) preference between the groups were compared using a repeated measures ANOVA, with oxytocin vs. saline as the independent variables, and test day as the repeated measure.

\section{RESULTS}

\section{Study I}

During preconditioning, animals spent significantly more time in one compartment compared with the other, and this was the basis for determining preferred or nonpreferred side (mean $=-399.8, d f=11$, paired $t$ value $-6.37, p=0.0001$ ). Black and white sides of the box were roughly equally preferred by the animals (seven and five animals, respectively). In half of the animals the nonpreferred side was paired with oxytocin (treatment group) and in the other half this side was paired with saline (control group). The preferred side of the box was paired with saline in both groups. The data from one animal (control group) was excluded from the analysis because this animal exhibited abnormal behavior (almost no movement) on the testing day, and the animal's place preference values for the same day fell 3.5 standard deviations away from the mean of the group. Following conditioning, there was a change in place preference in both groups of animals (repeated measures ANOVA effect of time, $F_{(1,9)}=23.9, p=$ 0.0009 ), and there was a significant effect of treatment (repeated measures ANOVA effect of treatment, $F_{(1,9)}=$ 9.0, $p=0.014)$. Whereas saline-treated animals decreased their preference toward a non-preference to either side (mean $=-132.4, \mathrm{SE}=111.6$ ) oxytocin-treated animals switched their preference to oxytocin paired side $($ mean $=224, \mathrm{SE}=113$ ) (see Figure 1). Post hoc comparisons revealed that there was a significant difference in place preference between the treatment groups on postconditioning day (Newman-Keuls, $p=0.035$ ),

\section{Study I}

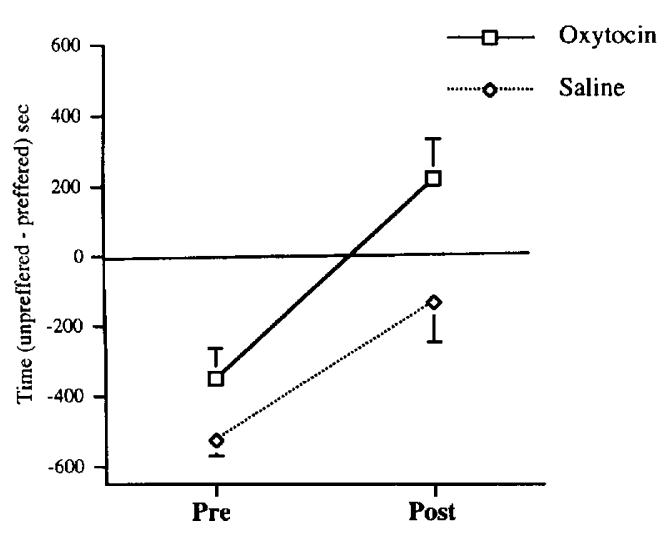

Figure 1. Effects of oxytocin, paired with the initially nonpreferred compartment, on CPP. The saline group received saline in both compartments. Note: whereas animals treated with saline lost preference (habituated), oxy tocin treated animals reversed their preference, developing preference to the oxytocin paired compartment. 


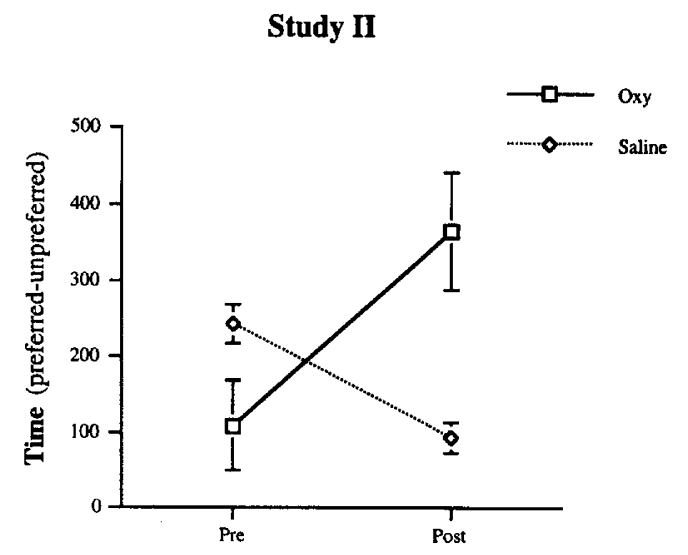

Figure 2. Effects of oxytocin, paired with the initially preferred compartment, on CPP. The saline group received saline in both compartments. Oxytocin-treated animals further enhanced their preference to the oxytocin paired compartment.

while there was no difference between the groups on the preconditioning day (Newman-Keuls, $p=0.25$ ).

\section{Study II}

Since in Experiment 1 we observed a change in the same direction in both groups (away from the nonpreferred compartment), the contribution of nonspecific effects of habituation and loss of place preference, as a result of time spent in the testing apparatus, could not be ruled out. To isolate the effects of oxytocin from the possible effects of habituation and loss of place preference we performed a second study pairing oxytocin with the initially preferred side of the shuttle box. The preferred side of the box was paired with oxytocin or saline, as described above. There was no significant dif-

Study III

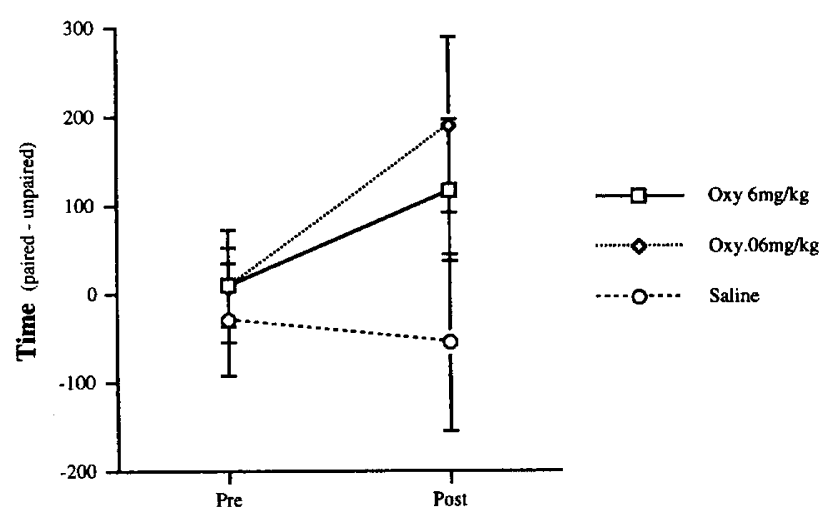

Figure 3. Effects of oxytocin and saline on CPP in a balanced design. Note the direction of change in both groups of animals treated with oxytocin as compared to saline-treated animals. ference in preference between oxytocin and saline groups prior to conditioning. Following conditioning, the saline-treated animals decreased their preference toward a nonpreference for either compartment, whereas oxytocin-treated animals further increased their preference for the oxytocin paired compartment (repeated measure ANOVA significant effect of interaction $F_{(1,9)}=$ $18.4, p=0.002$ ) (See Figure 2).

\section{Study III}

In the third experiment we attempted to establish a dose response relationship for the effects of oxytocin on place preference. Overall results show no significant effect of dose on place conditioning (repeated measures ANOVA, $F=1.92, d f=2, p=0.17$ for dose and $F=$ $0.9847, d f=2, p=0.39$ for interaction). However, since there was no apparent difference between two doses of oxytocin, the data for these groups was pooled and the effects reanalyzed. These results demonstrate that animals injected with oxytocin increased their preference after conditioning to the oxytocin paired side (repeated measure ANOVA effect of treatment $F_{(1,17)}=9.1, p=$ 0.007) (See Figure 3).

We have measured locomotor activity during the experiments to ascertain the possibility of animals changing their behavior (such as demonstrating freezing or exploratory behavior) following oxytocin administration. Presence of such change, unrelated to the motivational or hedonic aspects of the behavior, would confound the interpretation of our results. The activity of the animals during the conditioning phase did not differ between the oxytocin and saline groups, and remained constant with some day to day variations (Fig. 4 - only data from Study II is shown). Activity is ex-

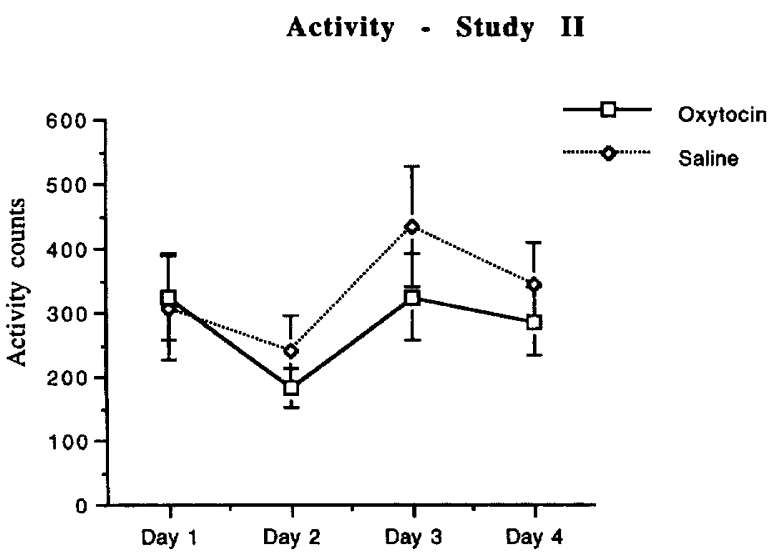

Figure 4. The effects of oxytocin and saline injections on locomotion during the eight conditioning days (four saline and four oxytocin). There was no significant difference between the groups on either day or overall. Only Study II data are shown for the purpose of illustration. 
pressed as photobeam breaks. There was no difference in activity in the oxytocin paired and saline paired compartments in either of the studies on the testing day. Overall, there was a trend for increased activity on the postconditioning day as compared to the preconditioning day (Study III, repeated measure ANOVA, effect of time $F_{(2,17)}=3.2, p=0.09$ ). However, there was no difference between the oxytocin and saline paired groups and there was no interaction effect between the pharmacological agent and the day of testing.

\section{DISCUSSION}

In the current studies we have demonstrated the effects of oxytocin on conditioned place preference in male rats. Whether it was paired with the initially nonpreferred compartment, the initially preferred compartment, or administered in a balanced manner, oxytocin caused an increase in preference for the compartment associated with the peptide. The results suggest that oxytocin has rewarding properties when administered subcutaneously in pharmacological doses. In contrast, control animals-paired with saline in both compartmentsprogressively lose their initial place preference and after eight days of exposure display little or no place preference, spending an equal amount of time in both sides of the shuttle box. The latter effects have previously been reported and interpreted as habituation to the apparatus over the eight days of conditioning (Trujillo et al. 1991). In view of this natural process we believe the finding of an enhancement of preference exhibited in the second experiment is especially convincing.

Since oxytocin was administered subcutaneously, we cannot ascertain whether this effect is mediated via peripheral or central mechanisms. We were unable to demonstrate a dose-response relationship using two oxytocin doses in the last experiment. Taken together, oxytocin treated animals changed their place preference similarly to the first two experiments, however there were no significant differences found when analyzed separately with a repeated measures ANOVA. The balanced design, a relatively small number of animals tested, and the higher variability displayed could account for the lack of an overall statistically significant effect. It should be noted, however, that in other CPP studies, dose response relationships were at times difficult to demonstrate, even for pharmacological agents with very strong rewarding properties like cocaine (Bardo et al. 1995).

The present findings have relevance for the behavioral effects of exogenously administered oxytocin. They may also have implications for the motivational milieu of internal states characterized by high endogenous oxytocin secretion or high oxytocinergic activity. Thus during maternal, sexual or affiliative behaviors, when central oxytocinergic activity is presumably high (Jirikowski 1992) the animal might associate general environmental cues with a positive hedonic state, rather than the specific behavioral activity (lactation, grooming, or coitus). While multiple, neurophysiological changes occur during copulation, oxytocin's ability to induce conditioned place preference might be one of the mechanisms that contributes to the development of CPP following sexual encounter (Oldenburger et al. 1992).

Although shifts in place preference, such as those observed in the present experiment for oxytocin, are typically interpreted as reflecting reinforcing properties of the agent under study, there are alternative explanations. First, this effect may reflect "anti-stress" or anxiolytic properties of oxytocin. Protective effects of exogenous oxytocin, and of physiological conditions associated with high oxytocin secretion (lactation), have been reported in behavioral and neuroendocrinological studies of stress (Grassi and Drago 1993; Lightman 1992; Panksepp 1992). Peripheral administration of oxytocin at the time of a stressor (such as exposure to the experimental conditions), could induce a less aversive internal state, reducing the overall total impact of stress, and appearing anxiolytic or protective. In the same vein, studies of MK-801 (NMDA receptor antagonist), showed that it induces CPP in animals suffering from inflammation, further supporting the notion that the decrease in aversive components of the experience could account for the development of CPP (Sufka 1994). It is therefore possible that the increase in time spent in the oxytocin-paired compartment reflects decreased anxiety or stress over aversive conditions inadvertently present in the testing condition, rather than overt reward (Carr et al. 1989). Although the change in hedonia for both of these circumstances is in a positive direction, there are important theoretical and practical distinctions between increased reward and decreased aversion.

While it is possible that decreased aversion might be involved in the present effects, several considerations suggest that this might be a less likely explanation. Animals were handled for six days prior to testing, followed by three days of exposure to the apparatus prior to conditioning. Therefore, they were very familiar with the experimenter and the testing situation, and showed no visible distress during testing. Additionally, saline animals showed a diminished preference after conditioning, suggesting that they were well-habituated by the time of testing. It seems unlikely that a decrease in aversion would produce such dramatic shifts in preference in such well-habituated animals. DiScala (1992) suggests that the existence of pre-conditioned aversion is important, for the ability of anxiolytic agents to induce CPP, but in our Study II, animals that were conditioned with oxytocin in the initially preferred compartment showed an even greater preference after conditioning. It seems, therefore, that such a robust effect would be 
more likely from a drug that produces reward than from one that is anxiolytic.

A second alternative explanation pertains to the reported amnestic effects of oxytocin. Studies on learning and memory suggest that oxytocin produces a general amnesia (Bohus 1980). As discussed by Carr et al. (1989), animals will show a place preference for an environment that is relatively more novel than another. In the present study, if oxytocin produced amnesia for the compartment with which it was paired, this may have caused that environment to be less familiar than the saline paired compartment, leading to a "noveltyinduced" place preference. It was also suggested that drugs that improve memory are more likely to produce $\mathrm{CPP}$, by strengthening the associative link (Carr et al. 1989). One might also interpret oxytoxin's effects on memory as secondary to its effects on motivational systems. Memory study paradigms routinely test animal memory of rewarding or aversive testing conditions. Oxytocin might affect memory of a given situation, by changing the hedonic or motivational milieu, thus altering its relative value or importance for memorization.

To summarize, the present results demonstrate that oxytocin produces a preference for the environment with which it is repeatedly associated. Although we feel that the most likely explanation for the present results appear to be that oxytocin has rewarding properties, other explanations, including potential anti-aversive or anti-stress properties, or amnestic effects cannot be ruled out. Future studies should utilize different experimental paradigms in an attempt to distinguish among these three possibilities.

\section{ACKNOWLEDGMENTS}

The authors wish to acknowledge the support of MH 00427 and MH 45232 to EAY, a Young Investigator Award from the National Alliance for Research on Schizophrenia and Depression to KAT, and NIDA 5R01DA02265/18 grant to HA.

\section{REFERENCES}

Agren G, Lundeberg T, Uvnas-Moberg K, Sato A (1995): The oxytocin antagonist 1-deamino-2-D-Tyr-(Oet)-4-Thr-8Orn-oxytocin reverses the increase in the withdrawal response latency to thermal, but not mechanical nociceptive stimuli following oxytocin administration or massage-like stroking in rats. Neurosci Lett 187:49-52

Arletti R, Benelli A, Bertolini A (1990): Oxytocin inhibits food and fluid intake in rats. Physiol Behav 48:825-830.

Bardo MT, Rowlett JK, Harris MJ (1995): Conditioned place preference using opiate and stimulant drugs: a metaanalysis. Neurosci Biobehav Rev 19:39-51

Bohus B (1980): Vasopressin, oxytocin and memory: effects on consolidation and retrieval processes. Acta Psychiatr Belg 80:714-720

Buijs RM (1978): Intra- and extrahypothalamic vasopressin and oxytocin pathways in the rat. Pathways to the limbic system, medulla oblongata and spinal cord. Cell Tissue Res 192:423-435

Buijs RM, Swaab DF (1979): Immuo-electron microscopical demonstration of vasopressin and oxytocin synapses in the limbic system of the rat. Cell Tissue Res 204:355-365

Burbach JP, Adan RA, de Bree FM (1992): Regulation of oxytocin gene expression and forms of oxytocin in the brain. In Pedersen CA, Calwell JD, Jirikowski GF, Insel TR (eds), Oxytocon in Maternal, Sexual, and Social Behaviors. Arlington, Virginia, Ann N Y Acad Sci, pp 1-492

Caldwell JD (1992): Central oxytocin and female sexual behavior. Ann N Y Acad Sci 652:166-179

Carr JD, Fibiger HC, Phillips AG (1989): Conditioned place preference as a measure of drug reward. In Liebman JM, Cooper SJT (eds), Neuropharmacological Basis of Reward. Oxford, Oxford University Press, pp 265-320

Carter CS, Williams JR, Witt DM, Insel TR (1992): Oxytocin and social bonding. Ann N Y Acad Sci 652:204-211.

de Wied D (1978): Neuropeptides: effects on motivation, learning and memory precesses. In Carenza L (ed), Clinical Psychoneuroendocrinology in Reproduction. London, Academic Press, pp 15-24

de Wied D, Elands J, Kovacs G (1991): Interactive effects of neurohypophyseal neuropeptides with receptor antagonists on passive avoidance behavior: mediation by a cerebral neurohypophyseal hormone receptor? Proc Natl Acad Sci U S A 88:1494-1498

de Wied D, Gaffori O, Burbach JP, Kovacs GL, van Ree JM (1987): Structure activity relationship studies with C-terminal fragments of vasopressin and oxytocin on avoidance behaviors of rats. J Pharmacol Exp Ther 241:268-274

Di Scala G, Oberling P, Rocha B, Sandner G (1992): Conditioned place preference induced by Ro 16-6028, a benzodiazepine receptor partial agonist. Pharmacol Biochem Behav 41:859-862

Grassi M, Drago F (1993): Effects of oxytocin on emotional stress and stress-induced gastric lesions. J Physiol Paris $87: 261-264$

Insel TR, Shapiro LE (1992a): Oxytocin receptor distribution reflects social organization in monogamous and polygamous voles. Proc Natl Acad Sci U S A 89:5981-5985

Insel TR, Shapiro LE (1992b): Oxytocin receptors and maternal behavior. Ann N Y Acad Sci 652:122-141

Insel TR, Winslow JT (1991): Central administration of oxytocin modulates the infant rat's response to social isolation. Eur J Pharmacol 203:149-152

Jirikowski GF (1992): Oxytocinergic neuronal systems during mating, pregnancy, parturition, and lactation. Ann NY Acad Sci 652:253-270

Keverne EB, Kendrick KM (1992): Oxytocin facilitation of maternal behavior in sheep. Ann N Y Acad Sci 652:83-101

Kovacs GL, Bohus B, Versteeg DH, de Kloet ER, de Wied D (1979): Effect of oxytocin and vasopressin on memory consolidation: sites of action and catecholaminergic correlates after local microinjection into limbic-midbrain structures. Brain Res 175:303-314 
Leckman JF, Goodman WK, North WG, Chappell PB, Price LH, Pauls DL, Anderson GM, Riddle MA, McSwigganHardin M, McDougle CJ, et al. (1994): Elevated cerebrospinal fluid levels of oxytocin in obsessive-compulsive disorder. Comparison with Tourette's syndrome and healthy controls. Arch Gen Psychiatry 51:782-792

Lightman SL (1992): Alterations in hypothalamic-pituitary responsiveness during lactation. Ann N Y Acad Sci 652:340-346

Mazurek MF, Beal MF, Bird ED, Martin JB (1987): Oxytocin in Alzheimer's disease: postmortem brain levels. Neurology 37:1001-1003

Oldenburger WP, Everitt BJ, de Jonge FH (1992): Conditioned place preference induced by sexual interaction in female rats. Horm Behav 26:214-228

Panksepp J (1992): Oxytocin effects on emotional processes: separation distress, social bonding, and relationships to psychiatric disorders. Ann N Y Acad Sci 652:243-252

Papp M, Willner P, Muscat R (1991): An animal model of anhedonia: attenuation of sucrose consumption and place preference conditioning by chronic unpredictable mild stress. Psychopharmacology 104:255-259

Sarnyai Z, Kovacs GL (1993): Role of oxytocin in the neuroadaptation to drugs of abuse. Psychoneuroendocrinology 18:85-117
Sufka KJ (1994): Conditioned place preference paradigm: a novel approach for analgesic drug assessment against chronic pain. Pain 58:355-366

Tribollet E, Barberis C, Jard S, Dubois-Dauphin M, Dreifuss JJ (1988): Localization and pharmacological characterization of high affinity binding sites for vasopressin and oxytocin in the rat brain by light microscopic autoradiography. Brain Res 442:105-118

Tribollet E, Dubois-Dauphin M, Dreifuss JJ, Barberis C, Jard $S$ (1992): Oxytocin receptors in the central nervous system. Distribution, development, and species differences. Ann N Y Acad Sci 652:29-38

Trujillo KA, Belluzzi JD, Stein L (1991): Naloxone blockade of amphetamine place preference conditioning. Psychopharmacology (Berl) 104:265-274

Van Erp AM, Kruk MR, Semple DM, Verbeet DW (1993): Initiation of self-grooming in resting rats by local PVH infusion of oxytocin but not alpha-MSH. Brain Res 607 : 108-112

van Erp AM, Kruk MR, Veening JG, Roeling TA, Meelis W (1995): Neuronal substrate of electrically induced grooming in the PVH of the rat: involvement of oxytocinergic systems? Physiol Behav 57:881-885 